\title{
La Administración electrónica \\ en la Unión Europea
}

\author{
Raquel Gómez Díaz \\ Yolanda Martín González \\ Universidad de Salamanca (España)
}

\section{Resumen}

Las tecnologías de la información y la comunicación ofrecen amplias posibilidades a la Unión Europea para mejorar y simplificar su propia administración interna en aras de una mayor transparencia y acceso a su información. Al tiempo, permiten una mayor eficacia en el servicio a los ciudadanos y a las empresas al propiciar una interacción más explícita entre las instituciones y los organismos públicos. Como primer paso hacia la gestión electrónica de la Administración eurocomunitaria, la Unión Europea adoptó en 2002, dentro del plan de acción eEurope 2005: una sociedad de la información para todos, la iniciativa eGovernment. El objetivo de este artículo es dar a conocer el plan estratégico diseñado por la Comisión para este primer quinquenio. Para ello, se analiza cómo se está llevando a cabo el proceso de transformación de una Administración presencial a una virtual y cómo el acceso electrónico a los servicios administrativos de la Unión Europea contribuye a una mayor democracia y transparencia en la Administración eurocomunitaria.

Palabras clave: Gestión de la información. Unión Europea. Administración electrónica.

\section{Resumen}

The new technologies of the information and the communication offer to the European Union wide possibilities to improve and simplify their own internal administration in order to provide an increased transparency and a better access to its information. At the same time, they allow an improved effectiveness in their service to the European citizens and companies, and also enable a better interaction between the EU institutions and the public organisms of the Member States. As a first step towards the electronic administration in Europe, the European Union adopted in 2002 the initiative eGovernment, framed into the plan of action eEurope 2005: a society of the information for all. The goal of this paper is to analyze the strategic plan designed by the European Commission for this first five-year period.

Scire. $14: 1$ (en.-jun. 2008) 105-110. ISSN 1135-3716. 
It will be analyzed how the Comission is carrying out a transformation process into a virtual administration, and how electronic access to the government services in the Union contributes to strengthen democracy and transparency in the government of the European Union.

Keywords: eGovernment. European Union. Electronic information. Information management.

\section{Introducción}

Las tecnologías de la información y la comunicación (TIC) suponen una nueva oportunidad de mejora en las relaciones entre el ciudadano y la Administración. Si pensamos en una extensión tan amplia como la de la Unión Europea, sin duda alguna el hecho de que los ciudadanos puedan utilizar métodos no presenciales para relacionarse con la Administración será una gran oportunidad para igualar las posibilidades de acceso a la información y a los servicios que Europa puede prestar a sus ciudadanos.

Administración electrónica, e-Administración, Administración virtual y Ciberadministración (Pinto 2004) son las distintas denominaciones que recibe la forma de gestión pública donde las relaciones entre los ciudadanos y la Administración se producen gracias a las nuevas tecnologías, en especial a Internet.

A medida que las administraciones públicas han ido incorporando la tecnología informática, se han ido produciendo cambios significativos en la manera de prestar los servicios que van desde el propio diseño de los mismos hasta la manera en que se establece la relación con los administrados, pasando por los nuevos formatos de los documentos, así como en los horarios de disponibilidad de dichos servicios.

La Administración electrónica se ha hecho presente a través de una serie de servicios públicos en formato electrónico (pago de impuestos, descarga de formularios, notificaciones de cambio de domicilio...), y la principal novedad que estos ofrecen es que no están atados a un lugar físico ni sujetos a unos horarios concretos, lo cual, teniendo en cuenta la extensión de UE, supondrá una gran ventaja para los eurociudadanos. Así conseguimos que cualquier administrado pueda acceder desde el lugar que más le convenga (la oficina, su hogar...) y en el horario que desee, lo que se denomina 24 × 7 x 365 (24 horas al día, 7 días a la semana, 365 días al año), de modo que los usuarios ganan en flexibilidad, rapidez y comodidad. Además de a los usuarios, esta nueva forma de trabajo también beneficia a la propia Administración, ya que, a través de los foros de opinión, buzones de sugerencias, etcétera, le aporta información acerca de cómo el ciudadano percibe los servicios que se le ofrecen, y así consigue ser una Administración más cercana, transparente y dinámica. En otras palabras, las tecnologías posibilitan un modo de participación ciudadana más democrático. 
La Administración virtual puede ser un estímulo para modificar el funcionamiento de la organización, ya que permite una mayor coordinación y flexibilidad, al tiempo que se comparte la información. Las TIC podrán facilitar el cambio, pero no solucionarán por sí solas todos los problemas de la Administración, ya que la aceptación de este tipo de gestión pasa necesariamente porque todos los ciudadanos tengan acceso a las tecnologías, las conozcan y las sepan usar. Además es necesario que los ciudadanos confíen en la seguridad de los sistemas, sobre todo en lo que a privacidad de datos se refiere. La aceptación de la Administración electrónica requiere que el ciudadano sea el centro de la gestión, lo que implica un rediseño de la Administración y la racionalización de los procesos administrativos, lo que se denomina reingeniería de los procedimientos. La integración de todos los servicios de la Administración en un mismo sistema facilitará la tramitación de los expedientes, evitando duplicar la información solicitada al ciudadano y sacando un mayor partido a la información que las distintas partes de la Administración ya tienen y pueden compartir.

Para que la Administración electrónica sea verdaderamente eficaz tiene que garantizar el acceso de todos los ciudadanos, y, al menos por el momento, las nuevas tecnologías no están al alcance de todos, puesto que ni todos pueden acceder a ellas ni todos las saben utilizar. Es necesario que exista conexión entre las distintas administraciones, que los ciudadanos conozcan todas las posibilidades que les ofrece la Administración electrónica y que pierdan la desconfianza que les generan los medios electrónicos.

\section{La Ciberadministración eurocomunitaria}

La Unión Europea ha sido una de las primeras administraciones de carácter público que han aplicado las nuevas tecnologías a la gestión de las actividades administrativas. Desde hace algunos años, las autoridades eurocomunitarias han dispuesto programas de acción destinados a facilitar la relación entre el ciudadano y sus instituciones a través de herramientas informáticas que favorecen la agilización de los trámites burocráticos, al tiempo que pueden conocer el grado de satisfacción de los usuarios con los nuevos servicios.

La Comisión Europea fue la institución pionera en diseñar un plan de acción que se hizo público en el año 2000 cuando se dio a conocer el Libro blanco sobre la reforma de la Comisión. En dicho documento, y más concretamente en sus acciones 7, 8 y 9, se aboga por el establecimiento de una Administración electrónica europea. Un año más tarde, este Libro blanco fue secundado por la comunicación sobre la eComisión donde se ponía de manifiesto cuál iba a ser el plan estratégico a seguir por esta institución durante el quinquenio 2000-2005. El mencionado plan se enmarcaba en una iniciativa mucho más amplia denominada eEurope.

Scire. $14: 1$ (en.-jun. 2008) 105-110. ISSN 1135-3716. 
La finalidad principal de estos planes de acción y sus consiguientes iniciativas es la creación de una Unión Europea más democrática, más eficaz y, sobre todo, más transparente. Una Administración transparente genera la confianza de sus ciudadanos, y eso se consigue permitiéndoles el acceso a la información pública elaborada y custodiada por sus instituciones, simplificando los procedimientos administrativos, mejorando la calidad de la redacción de sus textos legislativos de manera que sean más comprensibles, y creando mecanismos de participación y consulta para los ciudadanos. Todo esto se lleva a cabo, además de con el uso de las nuevas tecnologías, con la puesta en marcha de una estrategia de información y comunicación que permita el conocimiento mutuo y el diálogo entre la Comunidad Europea y su ciudadanía.

Sin embargo, para que la UE sea una Administración totalmente virtual, se han de crear las infraestructuras necesarias y hay que contar con los recursos financieros y humanos adecuados. Se necesita, por tanto, que las otras instituciones europeas pongan también en marcha los mecanismos apropiados para la creación de la Administración electrónica, trabajando juntas de manera efectiva para conseguir responder a las expectativas de los ciudadanos a los que sirven.

\section{El plan de acción eEurope 2005 y la iniciativa eGovernment}

De forma gradual, la Unión Europea pretende convertirse en una Administración totalmente virtual, y para conseguirlo ha creado, dentro de su plan de acción eEurope 2005: una sociedad de la información para todos, una iniciativa denominada La Administración electrónica: eGovernment.

El plan de acción eEurope 2005 es el sucesor del plan eEurope 2002. Fue aprobado por el Consejo Europeo de Sevilla, celebrado en 2002. Su objetivo principal consiste en estimular el desarrollo de servicios, aplicaciones y contenidos, acelerando al mismo tiempo el despliegue de un acceso seguro a Internet de banda ancha. Uno de sus objetivos específicos es el establecimiento de unos servicios públicos modernos, entre los que se encuentra la puesta en marcha de una Administración en línea (eGovernment).

La implantación de unos servicios administrativos públicos en línea conlleva las siguientes acciones: la adopción por la Comisión de un marco de interoperabilidad (1) para facilitar la prestación de servicios paneuropeos de Administración electrónica a ciudadanos y empresas; servicios públicos interactivos y accesibles a todos mediante redes de banda ancha y accesos multiplataforma (teléfono, televisor, ordenador, etcétera); el equipamiento de todas las administraciones públicas con conexiones de banda ancha; el otorgamiento por vía electrónica de la mayoría de los contratos públicos; el fácil acceso de todos los ciudadanos a los puntos de conexión pública a Internet.

Scire. $14: 1$ (en.-jun. 2008) 105-110. ISSN 1135-3716. 
La e-Administración se ha convertido en una prioridad para la UE, que se muestra consciente de la necesidad de prestar servicios públicos más eficaces y de mejor calidad, reducir los plazos de espera de los usuarios y aumentar la transparencia en la gestión eurocomunitaria.

En el documento en el que se presentó la iniciativa eGovernment se define la Administración electrónica como "la utilización de las tecnologías de la información y las comunicaciones (TIC) en las administraciones públicas, asociada a cambios en la organización y nuevas aptitudes del personal. Su objetivo consiste en mejorar los servicios públicos, reforzar los procesos democráticos y apoyar a las políticas públicas". De este modo, se facilita la obtención de la información ofrecida por las instituciones públicas y las transacciones de los administrados, reduciendo los plazos de espera. Además, se favorece el establecimiento de una relación directa entre los administrados (ciudadanos y/o empresas) y los responsables. Gracias a herramientas tales como los foros en línea, los debates virtuales y las votaciones electrónicas, los ciudadanos pueden interrogar directamente a los responsables y expresar su opinión sobre las políticas públicas.

En la actualidad, los portales de Internet de ventanilla única se están convirtiendo en la norma para la prestación de servicios públicos a la población. Igualmente, la utilización de las TIC favorece las relaciones entre las administraciones locales, regionales y nacionales, lo cual reporta numerosos beneficios en cuanto a tiempo y dinero.

Sin embargo, y pese a que los resultados alcanzados hasta el momento son más bien positivos, la Comisión insiste en trabajar aún en una serie de aspectos cruciales para la generalización de la Administración electrónica, como son, entre otros, la reducción de la denominada "brecha digital", que impide a gran parte de la población acceder a la Administración en línea y a sus ya indudablemente ventajosos servicios, y la generación de confianza en estos nuevos servicios por parte del público.

\section{Conclusiones}

La utilización de las tecnologías propiciará una Administración más flexible y transparente, favoreciendo un uso más democrático de los recursos disponibles, a los que podrán acceder todos los ciudadanos.

La puesta en marcha de la Administración electrónica implica una serie de cambios tanto en la formación de los funcionarios y los usuarios, que deben aprender a dar y utilizar los nuevos servicios, como en las infraestructuras, ya que es necesario garantizar el acceso en buenas condiciones de todos los miembros de la Unión Europea.

Por otra parte, la consecución de una democracia electrónica por parte de la Unión está ya suponiendo la transformación de prácticas de trabajo, provisión de

Scire. $14: 1$ (en.-jun. 2008) 105-110. ISSN 1135-3716. 
servicios, tiempos de respuesta en la gestión pública, etcétera, y, por supuesto, el establecimiento de nuevas relaciones entre los gobiernos y la sociedad, así como nuevos modos de participación y manifestación de la voluntad de los ciudadanos en los distintos procedimientos de la Administración del Estado.

Los informes realizados hasta ahora sobre el éxito o fracaso alcanzado con el plan de acción eEurope 2005 son alentadores, en particular en lo que se refiere a la Administración electrónica. El porcentaje de servicios administrativos básicos totalmente accesibles en línea pasó del $17 \%$ al $43 \%$ entre octubre de 2001 y octubre de 2003. No obstante, aún existen aspectos susceptibles de mejora, ya que hay importantes discrepancias entre los distintos servicios ofertados por las administraciones de los Estados miembros.

\section{Notas}

(1) Por interoperabilidad se entiende la capacidad que tienen dos programas (un cliente y un servidor, por ejemplo) para intercambiar e interpretar sus datos correctamente.

\section{Referencias}

La Administración electrónica y los archivos: amenazas y oportunidades para la archivística. Jornada técnica (2003). Toledo: Junta de Comunidades de Castilla la Mancha, 2003.

eEurope 2005: una sociedad de la información para todos (plan de acción que se presentará con vistas al Consejo Europeo de Sevilla, 21-22 de junio de 2002). http://europa.eu.int/ information_society/eeurope/2002/news_library/documents/eeurope2005/ eeurope2005_es.pdf (2006-06-30).

Europe Digital Content on the Global Networks. http://www.cordis.lu/econtent/ (200606-30).

Europe's Information Society: Thematic Portal. http://europa.eu.int/information_society/ eeurope/2005/index_en.htm (2006-06-30).

Gómez Camarero, Carmen (2003). Las nuevas formas de comunicación de la Administración con el ciudadano. // Anales de Documentación. 6 (2003) 109-119.

MAP (2000). Libro blanco para la mejora de los servicios públicos: una nueva Administración al servicio de los ciudadanos. Madrid: MAP, 2000.

Martín Rodrigo, Tomás (2000). Planteamientos para una Administración electrónica. // Tecnimap 2000. VI Jornadas sobre Tecnologías de la Información para la Modernización de las Administraciones Públicas (Cáceres, 23, 24 y 25 de octubre de 2000).

Pinto Molina, María; Gómez Camarero, Carmen (2004). La Ciberadministración en la sociedad de la información: retos y perspectivas. Gijón: Trea, 2004.

Tricas Lamana, Fernando (2002). E-servicios, un reto para las administraciones públicas: la transformación de procesos. // VII Congreso Internacional del CLAD sobre la Reforma del Estado y de la Administración Pública (Lisboa, 8-11 oct. 2002). http://unpan1.un.org/intradoc/groups/public/documents/CLAD/clad0043808.pdf (2006-06-30). 